\title{
Historia de Embarazos en Estudiantes de Programas de Salud en una Universidad Pública del Caribe Colombiano
}

\author{
Shyrley Díaz Cárdenas ${ }^{a}$, Farith González Martínez ${ }^{\mathrm{b}}$, Ketty Ramos Martínez
}

a Odontóloga, Magíster en

Salud Pública. Profesora

Catedrática, Departamento de Proyección Social,

Facultad de Odontología, Universidad de Cartagena (Colombia).

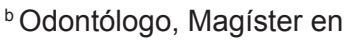
Salud Pública. Profesor Auxiliar, Departamento de Investigación, Facultad de Odontología, Universidad de Cartagena (Colombia). ' Odontóloga, Magíster en Salud Pública. Profesora Asistente, Departamento de Proyección Social, Facultad de Odontología. Universidad de Cartagena (Colombia).

Correspondencia:

Shyrley Díaz Cárdenas,

Departamento de

Investigaciones, Universidad de Cartagena, Facultad de Odontología, Campus de la Salud, Barrio Zaragocilla, Cartagena de Indias, Bolívar, Colombia. Telf.: 5756698172 , ext. 110, correo electrónico: shyrley77@hotmail.com

Recibido el 4 de mayo de 2010.

Aceptado para su publicación el 25 de agosto de 2010.

\begin{abstract}
RESUMEN
Objetivo. Describir la historia de embarazos y su relación con los conocimientos, actitudes y prácticas en estudiantes universitarias de programas de la salud en la Universidad de Cartagena.

Diseño. Descriptivo transversal.

Emplazamiento. Municipio de Cartagena de Indias-Colombia.

Participantes. Muestra de 231 estudiantes universitarias, a través de un muestreo probabilístico proporcional al programa y año de estudio en curso.

Mediciones principales. Se completó un cuestionario estructurado con las siguientes variables: historia de embarazos, tipo de relación de pareja, circunstancias y consecuencias, conocimientos, actitudes y prácticas sobre los anticonceptivos y algunas variables demográficas. Para las estimaciones se utilizaron las proporciones y la relación se evaluó mediante un análisis bivariado, aplicándose la prueba $X^{2}$ para encontrar la significación estadística.

Resultados. El 7,4\% de las encuestadas manifestó haber estado embarazada al menos una vez durante sus estudios. En cuanto a las prácticas, el método de anticoncepción más utilizado fue el preservativo (condón) con el 17,8\%. Al relacionar la historia de embarazos con las variables demográficas y las prácticas, se observó significación con la edad $(p=$ $0.006)$ y con el estado civil $(p=0.000)$, con el anticonceptivo más utilizado $(p=0.000)$ y con el conocimiento sobre los días fértiles dentro del ciclo menstrual $(p=0.005)$.

Conclusiones. Aunque esta población tiene fácil acceso a la información relacionada con los embarazos no deseados, se encontró una importante frecuencia de conocimientos no adecuados, prácticas poco seguras y actitudes que deforman la realidad de la anticoncepción de las estudiantes universitarias, generando cambios en sus proyectos de vida como profesionales.
\end{abstract}

Palabras Clave. Embarazo, Anticoncepción, Estudiantes Universitarios.

\section{ABSTRACT}

History of Pregnancies in Student of Health Programs in a Public University in the Colombian Caribbean

Objective. To describe the history of pregnancies and its relationship to the knowledge, attitudes and practices of university students in health programs. University of Cartagena.

Design. Cross-sectional.

Location. Municipality of Cartagena de Indias-Colombia.

Participants. Sample of 231 college students, through probability proportional sampling according to the program and year of study course.

Main measures. A structured questionnaire was completed with the following variables: history of pregnancies, type of relationship, circumstances and consequences, knowledge of, attitudes to and contraception practices, plus demographic variables. For estimates we used the proportions and the relationship was evaluated by bivariate analysis, applying the $\mathrm{X} 2$ test to determine statistical significance.

Results. Seven point four percent of the respondents reported having been pregnant at least once during their course of studies. The most commonly used method of contraception was the condom (condom) with $17.8 \%$ of students using this method. On correlating the history of pregnancies with demographic variables and practices, significance was observed with age $(P=0.006)$ and marital status $(P=0.000)$, with the most commonly used contraceptive $(P=0.000)$ and with the knowledge of fertile days within the menstrual cycle $(P=0.005)$

Conclusions. Although this population has easy access to information on unwanted pregnancies, there was a significant frequency of inadequate knowledge, unsafe practices and attitudes that distort the university students' reality of contraception and bring about changes in their life projects as professionals.

Keywords. Pregnancy, Contraception, University Students. 


\section{INTRODUCCIÓN}

La adolescencia es un periodo de cambio, crecimiento y también desequilibrio a nivel físico, psicológico, social y sexual, el cual implica también nuevas formas de exploración y de participación en la actividad sexual según la OMS. Las primeras relaciones sexuales en esta etapa son de suma importancia por la poca experiencia que se tiene al respecto, lo cual puede llevarlos a enfrentar problemas como el embarazo no planificado. Los embarazos no deseados son especialmente comunes en adolescentes, mujeres solteras y mayores de 40 años, sin embargo, también se dan en otros grupos de edad. Sus consecuencias no solo afectan a las mujeres, sino también a los hombres, las familias y a toda la sociedad ${ }^{1}$.

Las estudiantes universitarias, jóvenes en edad fértil, parecen no escapar a esta situación, encontrando así en muchos estudios que las adolescentes entre 15 y 19 años no utilizan ningún tipo de método anticonceptivo, a pesar de que una gran parte de ellas se inician en la vida sexual a temprana edad ${ }^{2}$.

En Colombia existe un conocimiento, por lo menos básico o elemental, de al menos un método anticonceptivo. Entre las mujeres adolescentes en unión libre el uso de métodos anticonceptivos es del 57 \% (Profamilia 2005, Colombia). La principal fuente de información respecto a métodos anticonceptivos son los profesores (32\%), tanto para hombres como para mujeres; en segundo lugar acuden a los amigos (19\%), sin embargo, en el caso de las mujeres es más importante la madre, frente a las instituciones de salud y otras fuentes especializadas $(5 \%)^{3}$

Según encuestas realizadas por Profamilia en el 2005 , cerca de 3.500 .000 de los colombianos son jóvenes entre los 15 y 19 años y aproximadamente la mitad de ellos ya han iniciado su vida sexual, y un $12,8 \%$ de estos la maternidad. Esta iniciación cada vez más temprana en los jóvenes de su vida sexual es debido a una maduración sexual temprana, la constante exposición a estímulos eróticos y la concepción errónea que se tiene de la sexualidad. A esto hay que sumarle el bajo nivel de comunicación entre padres e hijos acerca de temas sexuales ${ }^{4}$.

El objetivo de este estudio fue describir la historia de embarazos y su relación con los conocimientos, actitudes y prácticas en estudiantes de la Universidad de Cartagena, como una primera aproximación de esta situación en los programas del área de la salud, quienes reciben formación en educación sexual. Posteriormente, sus resultados deben encaminarse hacia la definición de acciones preventivas que propicien comportamientos sexuales responsables.

\section{MATERIAL Y MÉTODO}

El presente estudio es de tipo descriptivo transversal con enfoque cuantitativo. La población total para el estudio estuvo conformada por 935 estudiantes de sexo femenino de las diferentes facultades del área de la salud (de primer a quinto año de estudio en curso).

La población diana fueron todas las estudiantes matriculadas en algún programa del área de la salud de la Universidad de Cartagena en el segundo periodo académico de 2008. Se calculó una muestra a partir de una confianza del $95 \%$, un error del $5 \%$ y una frecuencia esperada del fenómeno objeto de estudio del $15 \%{ }^{5}$, obteniéndose 233 estudiantes, de los cuales dos no completaron el cuestionario (tasa de respuesta de 99,1\%). El método de muestreo utilizado fue probabilístico, de acuerdo a la distribución proporcional de los estudiantes en cada programa académico y año de estudio en curso. De esta forma se obtuvo una probabilidad conocida de los participantes en el estudio, de acuerdo con estas dos variables, las cuales se consideran como potenciales factores de influencia en los resultados. En este mismo sentido, el muestreo fue por reemplazo para sortear a las participantes que no se encontraban en el aula de clase en el momento de la aplicación del cuestionario.

Como criterios de selección se tuvieron en cuenta a estudiantes matriculadas en cualquier programa del área de la salud de la Universidad de Cartagena, entre primer y quinto año y se excluyeron a las estudiantes universitarias matriculadas en carreras semi-presenciales y estudiantes que estaban realizando internados.

Se diseñó un cuestionario estructurado, auto administrado, con 40 preguntas; 13 fueron dicotómicas y 27 politómicas, las cuales evaluaron las siguientes variables: historia de embarazos, tipo de relación de pareja, circunstancias y consecuencias en que ocurrió, conocimientos, actitudes y prácticas sobre el uso de anticonceptivos, y además se incluyeron algunas variables demográficas (edad, estrato socioeconómico, programa académico, año cursado y estado civil).

Para obtener la validez de apariencia y la plausibilidad teórica del instrumento, se realizaron dos 
sesiones de calibración con dos expertos en salud pública y luego se evaluaron las características del formulario a través de una prueba piloto, incluyéndose la comprensión de las preguntas, semántica, sintaxis, coherencia y pertinencia.

Previo a la recogida de la información, se tuvo en cuenta un consentimiento informado por escrito a partir de la normatividad internacional Helsinki modificación de Edimburgo $2000^{6}$ y resolución 8430 de 1993, Ministerio de Salud República de Colombia ${ }^{7}$. Las consideraciones éticas fueron necesarias para evitar violar la intimidad de los participantes a partir de los datos individuales obtenidos. Además el presente estudio fue considerado como "sin riesgo" a partir del concepto del comité de ética institucional de la Universidad donde se realizó el estudio.

Para el procesamiento y depuración de la información se utilizó el programa Microsoft Excel 2007®, y para el análisis se utilizó el programa STATA X®, versión para Windows 10.0, describiéndose las variables a través de medidas de tendencia central y dispersión. Para las estimaciones se utilizaron las proporciones, asumiendo intervalos de confianza del $95 \%$ y ajustados según la ponderación obtenida a través del marco muestral. La relación se evaluó mediante un análisis bivariado, aplicándose la prueba $X^{2}$ para encontrar la significación estadística.

\section{RESULTADOS}

Los participantes en el estudio fueron 231 estudiantes universitarias, excluyéndose dos cuestionarios que no fueron diligenciados completamente (tasa de respuesta del 99,1\%). El promedio de edad de los sujetos de estudio fue de 19,8 años (DE: 2,1). La facultad de Odontología aportó el $29,4 \%$, Enfermería el 28,6\%, Medicina el 19,5\%, Química farmacéutica el $15,2 \%$ y Química pura el $7,4 \%$. En cuanto al año en curso, el $56,3 \%$ estaban matriculadas entre primer y tercer año y el $43,7 \%$ entre cuarto y quinto año. Con respecto al estrato socioeconómico, la mayoría de las encuestadas pertenecían a los estratos socioeconómicos medio bajo y bajo con el $36,8 \%$ y $35,9 \%$ respectivamente,

\begin{tabular}{|c|c|c|c|}
\hline & $\begin{array}{c}\text { Frecuencia } \\
n=231\end{array}$ & $\begin{array}{c}\text { Porcentaje } \\
(\%)\end{array}$ & IC $95 \%$ \\
\hline \multicolumn{4}{|c|}{ Características del Embarazo } \\
\hline \multicolumn{4}{|c|}{ Ha tenido embarazos durante sus estudios } \\
\hline $\mathrm{Si}$ & 17 & 7,4 & $4-11$ \\
\hline No & 214 & 92,6 & $89-96$ \\
\hline \multicolumn{4}{|l|}{ Número de embarazos } \\
\hline Uno & 15 & 88,2 & $85-91$ \\
\hline Dos & 2 & 11,8 & $8-14$ \\
\hline \multicolumn{4}{|l|}{ La condición fue planificada } \\
\hline $\mathrm{Si}$ & 0 & 0,0 & - \\
\hline No & 17 & 100 & $98-102$ \\
\hline \multicolumn{4}{|c|}{ Embarazos con relación de pareja estable } \\
\hline & 17 & 100 & $98-102$ \\
\hline No & 0 & 0,0 & - \\
\hline \multicolumn{4}{|c|}{ Circunstancias del embarazo } \\
\hline Efectos del alcohol & 3 & 17,6 & $14-20$ \\
\hline Sobriedad & 14 & 82,4 & $79-85$ \\
\hline \multicolumn{4}{|c|}{ Edad de inicio de relaciones sexuales } \\
\hline $14-17$ & 7 & 41,2 & $15-66$ \\
\hline $18-21$ & 10 & 58,8 & $33-85$ \\
\hline \multicolumn{4}{|c|}{ Consecuencias del embarazo } \\
\hline Deserción estudiantil & 4 & 23,5 & $20-26$ \\
\hline Pérdida parcial de la salud & 5 & 29,4 & $26-32$ \\
\hline Ninguna & 8 & 47,1 & $44-50$ \\
\hline
\end{tabular}

Tabla 1. Aspectos relacionados con el Embarazo en estudiantes universitarias de programas de la Salud. Cartagena. 


\begin{tabular}{|c|c|c|c|c|c|}
\hline \multirow[b]{2}{*}{ V. Demográficas } & \multicolumn{2}{|c|}{ Historia de embarazo } & \multicolumn{2}{|c|}{ No historia de embarazo } & \multirow[b]{2}{*}{ Valor $\mathbf{P}$} \\
\hline & $\begin{array}{c}\text { Frecuencia } \\
n=17\end{array}$ & $\begin{array}{l}\text { Porcentaje } \\
\text { (\%) }\end{array}$ & $\begin{array}{c}\text { Frecuencia } \\
n=214\end{array}$ & Porcentaje (\%) & \\
\hline \multicolumn{6}{|l|}{ Edad } \\
\hline 20 a 24 & 14 & 6,1 & 102 & 44,2 & $0.006^{*}$ \\
\hline 16 a 19 & 3 & 1,3 & 112 & 48,5 & \\
\hline \multicolumn{6}{|l|}{ Programa } \\
\hline Odontología & 7 & 3,0 & 61 & 26,4 & 0.08 \\
\hline Enfermería & 8 & 3,5 & 58 & 25,1 & \\
\hline Medicina & 0 & 0,0 & 45 & 19,5 & \\
\hline Química farmacéutica & 2 & 0,8 & 33 & 14,3 & \\
\hline Química pura & 0 & 0,0 & 17 & 7,4 & \\
\hline \multicolumn{6}{|l|}{ Año en curso } \\
\hline Cuarto a Quinto año & 9 & 3,9 & 92 & 39,8 & 0.42 \\
\hline Primer a Tercer año & 8 & 3,5 & 122 & 52,8 & \\
\hline \multicolumn{6}{|l|}{ Estrato socioeconómico } \\
\hline Muy bajo- bajo & 7 & 3,0 & 117 & 50,7 & 0.28 \\
\hline Medio bajo- muy alto & 10 & 4,3 & 97 & 42,0 & \\
\hline \multicolumn{6}{|l|}{ Estado civil } \\
\hline Unión libre/casada & 6 & 2,6 & 1 & 0,4 & $0.000^{*}$ \\
\hline Soltera & 11 & 4,8 & 213 & 92,2 & \\
\hline Total & 17 & 7,4 & 214 & 92,6 & \\
\hline
\end{tabular}

Tabla 2. Relación entre historia de embarazos y variables demográficas en estudiantes universitarios de programas de la salud. Cartagena. * Estadísticamente significativo.

mientras que las estudiantes en el estrato medio alto, alto y muy alto solo aportaron un $9,5 \%$. Con respecto al estado civil, el $97 \%$ de las encuestadas eran solteras en el momento del estudio.

Con relación a la ocurrencia de embarazos, el 7,4\% (IC 95\%: 4-11) de las encuestadas manifestó haber estado en esta condición al menos una vez durante sus estudios, y de éstos se llevaron a termino completo un solo embarazo en la mayoría de los casos $(82,2 \%)$. De estos casos, todos fueron no planificados producto de una relación estable. El rango de edad más frecuente de inicio de relaciones sexuales fue entre 18 y 21 años con el 58,8\% (IC 95\%: 33-85). En cuanto a las circunstancias del embarazo, sólo el 17,6\% (IC 95\%: 14-20) manifestó haber estado bajo los efectos del alcohol, y como consecuencia del embarazo se produjeron problemas de salud en el $29,4 \%$ y deserción estudiantil temporal en el $23,5 \%$ (tabla 1 ).

En cuanto a los conocimientos, actitudes y prácticas de las participantes con respecto al uso de anticonceptivos, el más utilizado fue el preservativo (condón) con el 17,8\% (IC 95\%: 13-23), que además actúa como método preventivo contra las Infecciones de transmisión sexual simultáneamente, seguido de la píldora con el 9,5\% (IC 95\%: 6-13). Con respecto al origen de la información que obtienen sobre planificación familiar, el 44,6\% (IC 95\%: 38-51) manifestó que la han recibido de profesores durante su carrera universitaria. Por otra parte, las encuestadas que reportaron embarazos informaron que se abastecen de las farmacias en el 35,5\% (IC 95\%: 29-42) y de las Entidades Promotoras de Salud (EPS) en el 3,9\% (IC 95\%: 1-6). De acuerdo a los conocimientos de los participantes, se encontró que cerca de la mitad de los encuestados no tuvieron respuestas adecuadas en cuanto a los siguientes aspectos: "días fértiles dentro del ciclo menstrual”, sólo el 57,1\% (IC 95\%: 51-64) respondió en forma acertada; "cuál es el momento apropiado para tomar la temperatura basal como método anticonceptivo", el 57,6\% (IC 95\%: 51-64) respondió que todas las mañanas a la misma hora. Por otra parte, hubo una alta frecuencia de respuestas acertadas en otros aspectos como fueron: "hasta dónde debe llegar el condón para que sea efectivo como método anticonceptivo", el 94,4\% (IC 95\%: 91-97) de los encuestados manifestó que hasta su base; "el día propicio para la aplicación de los anticonceptivos inyectables", el 60,2\% (IC 95\%: 54-67) considera que el primer día de la menstruación.

De acuerdo a las percepciones sobre el uso de métodos anticonceptivos folklóricos ${ }^{8}$ solo el $50,7 \%$ (IC 95\%: 44-57) considera que existe riesgo de embarazo al utilizar el lavado de los genitales después 


\begin{tabular}{|c|c|c|c|c|}
\hline \multirow[b]{2}{*}{ Conocimientos, Actitudes y Prácticas } & \multicolumn{2}{|c|}{ Historia de embarazos } & \multirow[b]{2}{*}{$\begin{array}{c}\text { Total } \\
\mathbf{N}=231\end{array}$} & \multirow[b]{2}{*}{ Valor $p$} \\
\hline & $\begin{array}{l}\mathrm{Si}(\%) \\
N=17\end{array}$ & $\begin{array}{c}\text { No }(\%) \\
N=214\end{array}$ & & \\
\hline \multicolumn{5}{|l|}{ Anticonceptivo más utilizado } \\
\hline Condón & $4(9,8)$ & $37(90,2)$ & 41 & $0,000^{*}$ \\
\hline Píldora & $4(18,2)$ & $18(81,8)$ & 22 & \\
\hline Inyectable & $5(27,8)$ & $13(72,2)$ & 18 & \\
\hline Ritmo & $0(0,0)$ & $11(100)$ & 11 & \\
\hline DIU & $2(66,7)$ & $1(33,3)$ & 3 & \\
\hline \multicolumn{5}{|l|}{ Edad de inicio de relaciones sexuales } \\
\hline 14 a 17 & $7(12,7)$ & $48(87,3)$ & 55 & 0,4 \\
\hline 18 a 21 & $10(18,5)$ & $44(81,5)$ & 54 & \\
\hline \multicolumn{5}{|c|}{ Cuales son los días fértiles dentro el ciclo menstrual } \\
\hline Entre el $5^{\circ}$ y $10^{\circ}$ & $1(2,9)$ & $34(97,1)$ & 35 & $0,005^{*}$ \\
\hline Entre el $8^{\circ}$ y $19^{\circ}$ & $7(5,3)$ & $125(94,7)$ & 132 & \\
\hline Entre el $3^{\circ}$ y $15^{\circ}$ & $0(0,0)$ & $17(100)$ & 17 & \\
\hline No sabe & $9(19,1)$ & $38(80,9)$ & 47 & \\
\hline \multicolumn{5}{|c|}{ Momentos para utilizar el método de la temperatura basal } \\
\hline Todas las mañanas a la misma hora & $8(6,0)$ & $125(94,0)$ & 133 & 0,56 \\
\hline Una vez a la semana por las noches & $0(0,0)$ & $4(100)$ & 4 & \\
\hline Por las mañanas cada dos días & $0(0,0)$ & $5(100)$ & 5 & \\
\hline No sabe & $9(10,1)$ & $80(89,9)$ & 89 & \\
\hline \multicolumn{5}{|c|}{ Hasta donde debe cubrir el preservativo (condón) } \\
\hline Hasta su base & $17(7,8)$ & $201(92,2)$ & 218 & 0,57 \\
\hline La mitad del pene & $0(0,0)$ & $7(100)$ & 7 & \\
\hline No sabe & $0(0,0)$ & $6(100)$ & 6 & \\
\hline \multicolumn{5}{|l|}{ Día de inicio de anticonceptivos inyectables } \\
\hline Último día de la menstruación & $2(4,2)$ & $46(95,8)$ & 48 & 0,36 \\
\hline Primer día de la menstruación & $13(9,4)$ & $126(90,6)$ & 139 & \\
\hline No sabe & $2(4,5)$ & $42(95,5)$ & 44 & \\
\hline \multicolumn{5}{|c|}{ Existe riesgo de embarazo al lavarse los genitales después de una relación sexual } \\
\hline $\mathrm{Si}$ & $7(7,8)$ & $83(92,2)$ & 90 & 0,95 \\
\hline No & $8(4,2)$ & $109(57,7)$ & 189 & \\
\hline No sabe & $2(8,3)$ & $22(91,7)$ & 24 & \\
\hline \multicolumn{5}{|l|}{ Existe riesgo de embarazo en el coito de pie } \\
\hline $\mathrm{Si}$ & $6(7,3)$ & $76(92,7)$ & 82 & 0,96 \\
\hline No & $9(7,1)$ & $117(92,9)$ & 126 & \\
\hline No sabe & $2(8,7)$ & $21(91,3)$ & 23 & \\
\hline \multicolumn{5}{|c|}{ Existe riesgo de embarazo al tener relaciones sexuales con el periodo menstrual } \\
\hline $\mathrm{Si}$ & $8(9,6)$ & $75(90,4)$ & 83 & 0,57 \\
\hline No & $7(5,7)$ & $115(94,3)$ & 122 & \\
\hline No sabe & $2(7,7)$ & $24(92,3)$ & 26 & \\
\hline \multicolumn{5}{|c|}{ Existe riesgo de embarazo al tomar la píldora del día después } \\
\hline $\mathrm{Si}$ & $5(5,3)$ & $90(94,7)$ & 95 & 0,18 \\
\hline No & $12(10,2)$ & $106(89,8)$ & 118 & \\
\hline No sabe & $0(0,0)$ & $18(100)$ & 18 & \\
\hline
\end{tabular}

Tabla 3. Relación entre conocimientos, actitudes y prácticas de anticoncepción y la historia de embarazos en estudiantes universitarios de programas de la salud. Cartagena. * Estadísticamente significativo.

de la relación sexual, el 54,6\% (IC 95\%: 48-61) considera de riesgo la práctica del coito de pie, el 35,9\% (IC 95\%: 30-42) cree que no podrían quedar embarazadas al tener relaciones sexuales durante el periodo menstrual y, con respecto al uso de métodos anticonceptivos artificiales, el 41,1\% (IC 95\%:
35-48) no confía en la efectividad anticonceptiva de la píldora del día después.

En el análisis bivariado, al relacionar la historia de embarazos en la población encuestada con las variables demográficas, se observó significación 
con la edad, siendo las estudiantes mayores de 20 años las de mayor frecuencia de embarazos $(p=0.006)$. Además, hubo relación con el estado civil, observándose las estudiantes solteras con la mayor frecuencia de embarazos $(4,8 \% ; p=0.000)$. Con las demás variables, no se encontró relación estadísticamente significativa (tabla 2).

Al relacionar la historia de embarazos con los conocimientos, actitudes y prácticas sobre los métodos anticonceptivos, se encontró relación estadística con el anticonceptivo más utilizado, resaltándose que el preservativo (condón) es el método anticonceptivo de mayor uso en las estudiantes sin historia de embarazos $(p=0.000)$. También hubo relación estadísticamente significativa con el conocimiento sobre los días fértiles dentro del ciclo menstrual $(p$ $=0.005)$. Para las demás variables no se encontró significación (tabla 3).

\section{DISCUSIÓN}

A pesar de que los resultados del presente estudio se obtuvieron a partir de un cuestionario aplicado en un solo momento del tiempo, se consideran válidos y pueden ser útiles como una aproximación al fenómeno en estudio. Los sesgos de información se trataron de controlar con el diseño y posterior calibración de un cuestionario autoadministrado con respuestas comprensibles y pertinentes desde el contexto geográfico donde se encuentra ubicada la población diana (el Caribe Colombiano). Por otro lado, para evitar el sesgo de selección se eligió un muestro probabilístico de acuerdo a la distribución proporcional en que se encontraba distribuida la población objeto en algunas variables de importancia para el estudio (programa académico y año en curso). Por último, el sesgo de confusión se intentó prevenir a partir de la exclusión de variables consideradas extrañas que de alguna forma podrían competir con el fenómeno en estudio (historia de embarazos).

Según los resultados del presente estudio, el $7,4 \%$ de las encuestadas manifestaron haber tenido historia de embarazos durante su formación universitaria. Estos resultados son menores a los reportados por Ortiz ${ }^{5}$ en el $2003(15,0 \%)$, pero en esta población se incluyeron estudiantes de matemáticas y ciencias sociales, lo cual pudo haber influido en las diferencias.

Se supone que los estudiantes de los programas de la salud tendrían más acceso a información relacionada con métodos anticonceptivos que en otros programas universitarios no relacionados y que en ellos la frecuencia de historia de embarazo podría ser más baja. Hay que señalar que la prevalencia de este fenómeno en el presente estudio y el rango de edades de inicio de relaciones sexuales fue similar a los hallazgos reportados por la Encuesta Nacional de Demografía y Salud ${ }^{8}$ realizada en Colombia en 2005, donde exactamente el 7,4\% de jóvenes con educación superior ya son madres y la edad promedio de inicio de relaciones sexuales fue a los 18,4 años. En este sentido, es claro que el rol de madre adquirido, y no esperado durante el curso de su proyecto de vida como futura profesional, traería complicaciones para continuar con el curso y cumplimiento del mismo, además de convertirse en una posible fuente generadora de estrés por la sobrecarga de obligaciones, no solo académicas, sino las relacionadas con su nueva condición, las cuales comprometerían la calidad de vida de la nueva familia, y además conllevaría a la deserción estudiantil ${ }^{9}$, consecuencia ésta que se encontró en un porcentaje importante en el presente estudio, y que podría aumentarse a partir del avance del problema en mención.

Por otro lado, todas las estudiantes que manifestaron historia de embarazos afirman que esta condición no fue planificada. Estos hallazgos son similares a los reportados por Estupiñán ${ }^{10}$ en un estudio realizado con universitarias de la ciudad de Tunja-Colombia en el 2009, donde la gran mayoría lo reportó como una circunstancia no planeada. Con respecto a los factores relacionados con la aparición del embarazo en esta etapa de la vida, en el presente estudio solo una baja frecuencia de las participantes manifestó estar bajo los efectos del alcohol, contrario a lo reportado por Ospina ${ }^{11}$, quien concluyó que el consumo del alcohol es una de las causas más relevantes y relacionadas con la presencia de embarazos en estudiantes universitarios. En este sentido, es importante revisar el contexto que rodea a estas estudiantes para iniciar acciones que busquen controlar la presencia de factores de riesgo en esta población y la presencia del alcohol es uno de ellos.

Con respecto a los conocimientos sobre el uso de anticonceptivos, en cerca de la mitad de las encuestadas no se encontró un conocimiento apropiado para alguno de los aspectos valorados en el presente estudio (días fértiles dentro del ciclo menstrual y momento apropiado para tomar la temperatura basal como método anticonceptivo), lo cual coincide con Alves ${ }^{12}$ en el 2008, en Brasil, donde, además de evidenciar la necesidad de que los adolescentes universitarios conozcan más información sobre los métodos anticonceptivos, se sugiere que los profesores universitarios sean la fuente de esta información.

En cuanto al uso de métodos anticonceptivos, en el presente estudio el preservativo (condón) resultó 
ser el anticonceptivo más usado. Estos resultados son similares a los reportados por Vásquez ${ }^{13}$ en la Universidad del Valle, Colombia, explicándose probablemente por la difusión que ha tenido el preservativo desde los medios de comunicación en los últimos años, no solamente utilizado como anticonceptivo sino también como método para prevenir enfermedades de transmisión sexual, lo que hace que sea de doble propósito, así como de fácil acceso y bajo costo para los estudiantes. Con respecto a la percepción sobre prácticas anticonceptivas de riesgo, es importante destacar que casi la mitad de las universitarias son conscientes del peligro de quedar en embarazo al asumir prácticas naturales sin evidencias científicas que avalen su efectividad, lo que es reafirmado por Ospina ${ }^{11}$, además de que casi la mitad de las universitarias manifestó que no confían en la anticoncepción de emergencia. Esto coincide con lo encontrado por Tapia-Curiel ${ }^{14}$, en México en el 2008, donde la mitad de los jóvenes universitarios no conocen el uso correcto de la píldora post-coito y además muestran una actitud desfavorable hacia esta práctica.

A partir de los resultados obtenidos, se considera que pueden servir para la toma de decisiones desde la oficina de Bienestar universitario de la Universidad de Cartagena, lo que permitirá intervenir con éxito a las estudiantes en etapas tempranas del proceso educativo y evitar futuras consecuencias que pudiesen interferir con su continuidad dentro de su formación académica.

Se concluye entonces que, aunque los datos de historia de embarazos en el presente estudio son considerados de baja frecuencia en esta población, estos pueden representar una alarma inicial desde la salud pública, ya que las estudiantes universitarias en formación dentro del campo disciplinar del área de la salud tienen más fácil acceso a la información básica necesaria para evitar embarazos no planificados y aún así existen algunos aspectos dentro de los conocimientos, actitudes y prácticas que presentaron respuestas inadecuadas y pueden convertirse en riesgo al momento de tomar la decisión de la anticoncepción.

Se hace necesario entonces la implementación de intervenciones oportunas desde los primeros años de estudio de este grupo de estudiantes universitarias, para evitar que siga en aumento esta problemática y se convierta en la principal causa de la deserción temporal o definitiva de sus actividades académicas. Además, se recomienda incluir en un próximo estudio a estudiantes del sexo masculino, ya que el embarazo no es una condición vivida únicamente por la mujer.

\section{AGRADECIMIENTOS}

A la Universidad de Cartagena por el apoyo académico y administrativo durante la realización de este proyecto. A las estudiantes participantes en el presente estudio, las cuales diligenciaron el instrumento de recogida de datos acorde con la solicitud hecha por los investigadores.

\section{BIBLIOGRAFÍA}

1. Monterrosa A. Causas e implicaciones médico-sociales del embarazo en la adolescencia en Colombia. Rev Coloma Obstet Ginecol. 1998; 49(4):225-30.

2. Langer A, Espinosa $\mathrm{H}$. El embarazo no deseado: impacto sobre la salud y la sociedad en América Latina y el Caribe. Pan Am J Public Health. 2002; 11(3):194.

3. Pérez M, Padilla U, Serva L. Embarazo no planificado en estudiantes universitarias en control pre-natal. Medula. Journal of the Faculty of Medicine. 1993; 1(2):3-4.

4. Ceballos A, Campo A. Relaciones sexuales en estudiantes de un programa de medicina de la ciudad de Santa Marta. Rev Duazary. 2006; 2(2):110-4.

5. Ortiz C, Viafara G. Prevalencia de embarazo no deseado y modo de resolución en estudiantes universitarios: adolescentes y adultos jóvenes en Cali, Colombia en relación a la percepción de la calidad de educación sexual recibida. Rev Soc Chil Obstet Ginecol Infant Adolesc. 2003; 10(1):13-8.

6. World Medical Association (Declaration of Helsinki). Acceso el 25 de febrero de 2008. Disponible en: http://www.wma. net/s/ethicsunit/helsinki.htm.

7. Normas científicas, técnicas y administrativas para la investigación en salud. Resolución 008430. República de Colombia, Ministerio de Salud; 1993.

8. Encuesta Nacional de Demografía y Salud ENDS 2005. Profamilia. Resultados Departamento de Bolívar. Septiembre 21 de 2006. Acceso el 22 de enero de 2010. Disponible en www.minproteccionsocial.gov.co.

9. Rueda R, Parada A. Embarazo en adolescentes: El problema más grave en salud pública. Memorias Conferencia Academia Nacional de Medicina. Bogotá, Colombia; 10 de febrero de 2005.

10. Estupiñán M, Rodríguez L. Aspectos psicosociales en universitarias embarazadas. Rev. Salud Pública [online]. 2009; 11(6):988-98

11. Ospina J, Manrique F. Prácticas y comportamientos sexuales en estudiantes universitarios. Av Enferm. 2007; 25(2):101-11.

12. Alves S, López M. Uso de métodos anticonceptivos entre adolescentes universitarios. Rev Bras Enferm. 2008; 61(2):170-7.

13. Vásquez M, Argote L. La educación y el ejercicio responsable de la sexualidad en adolescentes. Rev Colom Med. 2005; 36(3s1):33-42.

14. Tapia-Curiel A. Anticoncepción de emergencia. Rev Med Inst Mex Seguro Soc. 2008; 46 (1):33-41. 